\title{
Características clínicas de fumantes atendidos em um centro de referên- cia na cessação do tabagismo
}

\section{Clinical characteristics of smokers seen in a smoking cessation reference center}

\author{
Suen K. K. Karen ${ }^{1}$, Maria Luisa Oliveira², Adriana I. de Pádua ${ }^{3}$, Fernanda Vieira ${ }^{4}$, José A. B. Martinez ${ }^{5}$
}

\begin{abstract}
RESUMO
Objetivo: Descrever características demográficas e clínicas de fumantes que procuraram atendimento em centro especializado na cessação de tabagismo. Métodos: Foram analisadas, retrospectivamente, as informações disponíveis em ficha clínica padronizada preenchida no momento do primeiro atendimento, de fumantes que procuraram o Ambulatório de Apoio ao Tabagista do Centro de Saúde Escola da Faculdade de Medicina de Ribeirão Preto da Universidade de São Paulo, no período de 18 meses. Resultados: O serviço foi procurado no período por 186 fumantes (135 mulheres), com idade de $50 \pm 12$ anos. A maioria (59\%) veio encaminhada de outros ambulatórios médicos, e o consumo apenas de cigarros industrializados foi relatado por $96 \%$. A idade de início do consumo regular de cigarros foi de $18 \pm 7$ anos. Experiências prévias frustradas de abandono foram relatadas por $83 \%$ dos fumantes, e o número médio de tentativas efetuadas foi de $3 \pm 2$. A maioria dos atendidos $(66 \%)$ exibia dependência à nicotina de grau alto ou muito alto, com escore médio do teste de Fargeström de $6 \pm 2$. Co-morbidades físicas foram relatadas em $62 \%$ e psiquiátricas em $24 \%$ dos casos, particularmente depressão (17\%). Conclusões: Os dados obtidos servem como embasamento de algumas necessidades a serem supridas por novos serviços de cessação do tabagismo a serem instalados em municípios de porte médio do Brasil e exterior.
\end{abstract}

Palavras chave: Tabagismo. Dependência de Tabaco. Dependência de Nicotina. Abandono do Uso de Tabaco. Epidemiologia Descritiva.

\section{Introdução}

O tabagismo é causa de inúmeras doenças, incluindo moléstias respiratórias, cardiovasculares e neoplasias. ${ }^{1,2}$ Os prejuízos do tabagismo não estão restritos aos seus usuários, uma vez que o fumo passivo pode ser importante fonte de malefícios. ${ }^{1}$ Os custos impostos pelo tabagismo ao sistema de saúde são enormes. ${ }^{3}$ Portanto, ações anti-tabágicas estão entre as intervenções de saúde pública mais custo-efetivas. ${ }^{4,5}$

Oitenta por cento dos fumantes refere o desejo de abandonar o tabaco, e a maioria consegue vencer
1. Acadêmica do curso médico da Faculdade de Medicina de Ribeirão Preto da Universidade de São Paulo (FMRP-USP).

2. Mestre em Psicologia. Psicóloga do Centro de Saúde Escola da FMRP-USP.

3. Doutora em Clínica Médica. Médica da Seção de Pneumologia do Hospital das Clínicas da FMRP-USP.

4. Psicóloga Clínica. Voluntária do Centro de Saúde Escola da FMRP-USP.

5. Professor Associado do Departamento de Clínica Médica da FMRP-USP.
Correspondência:

José Antônio Baddini Martinez / baddini@fmrp.usp.br Departamento de Clínica Médica da FMRP-USP.

Avenida Bandeirantes 3900. 14048-900. Ribeirão Preto. São Paulo

Artigo recebido em 11/06/2012 Aprovado para publicação em 13/07/2012 
a dependência sozinha, geralmente após duas ou três tentativas. ${ }^{4}$ Contudo, uma porção expressiva necessita de tratamento especializado para obter sucesso na empreitada. Desse modo, serviços de referência para tratamento de tabagistas vêm sendo crescentemente implantados em nível mundial. O objetivo deste estudo foi descrever as características demográficas e clínicas de fumantes que procuraram um ambulatório público, especializado em cessação de tabagismo, de uma cidade de porte médio do interior brasileiro. Tais características são discutidas em função de outros dados da literatura e quanto à sua possível influência no cuidado fornecido a esses sujeitos.

\section{Métodos}

Em fevereiro de 2009 foi instalado no Centro de Saúde Escola da Faculdade de Medicina de Ribeirão Preto da Universidade de São Paulo (CSE-FMRPUSP), ambulatório especializado para auxílio de fumantes no processo de abandono. Esse Serviço conta com médicos residentes, psicóloga e a supervisão de médico-assistente e docente, ligados à Divisão de Pneumologia do Departamento de Clínica Médica da FMRP-USP. O CSE-FMRP-USP atende a população da Distrital de Saúde Oeste do Município de Ribeirão Preto, prestando atendimento em nível primário e secundário a aproximadamente 250 mil habitantes.

No momento da criação do ambulatório foi desenvolvida ficha padrão, contendo informações tais como nome, data da consulta, nascimento, endereço, procedência, etc. Todos os fumantes consultados tiveram essa ficha preenchida pelo médico responsável. Todas as fichas de primeira consulta preenchidas foram arquivadas até tabulação e análise. No presente estudo foram incluídas fichas relativas aos primeiros 18 meses de funcionamento do serviço. Trata-se de um estudo descritivo, transversal, retrospectivo, em que foram incluídas informações relativas a todos os fumantes que procuraram o serviço e foram avaliados em consulta inicial no período de fevereiro de 2009 a agosto de 2010 .

As variáveis analisadas foram sexo, idade, procedência, tentativas prévias de parada, maior período sem fumar, coabitação com tabagistas, escala de Fargeström $^{6}$, co-morbidades físicas, co-morbidades psiquiátricas e uso de medicamentos. Os dados são apresentados de forma descritiva como frequências, médias e desvios-padrão. O projeto de pesquisa foi aprovado pelo Comitê de Ética da Instituição.

\section{Resultados}

Entre fevereiro de 2009 e agosto de 2010 foram atendidos 186 fumantes, cujas fichas foram disponíveis para análise (Tabela 1). Dos 186 fumantes $27 \%$ (51) eram homens e $73 \%$ (135) mulheres. A idade média foi $50 \pm 12$ anos. No tocante a distribuição, 28 fumantes (15\%) tinham idade entre 20 e 40 anos, $123(66 \%)$ entre 40 e 60 anos, e $35(19 \%)$ acima de 60 anos. A idade mínima foi de 20,2 e a máxima de 77,5 anos.

Em relação à procedência, $61(33 \%)$ sujeitos foram encaminhados por médicos atuantes no próprio CSE-FMRP, 25 (13\%) de ambulatórios do Hospital das Clínicas de Ribeirão Preto (HCRP), 23 (12\%) de outros ambulatórios externos do município, e 76 (41\%) procuraram o serviço por iniciativa própria, após conhecimento do programa por cartazes colocados no CSE-FMRP ou indicação de vizinhos e amigos. A informação não foi disponível em uma ficha $(0,5 \%)$.

A maioria dos fumantes relatava consumo apenas de cigarros $(178 ; 96 \%)$, enquanto sete $(4 \%)$ relatavam consumir cigarros de papel e diferentes produtos tabágicos, como charutos e cigarros de palha. Apenas um indivíduo $(0,5 \%)$ consumia exclusivamente cigarros de palha. A informação estava faltando para um indivíduo. Um número substancial de pessoas (86; $46 \%$ ) referiu a presença de fumantes em casa.

\section{Tabela 1}

Características clínicas dos fumantes em consulta inicial no programa de abandono de tabagismo.

\begin{tabular}{ll}
\hline Número de fumantes & 186 \\
Sexo & $\begin{array}{l}\text { Masculino: 51 (27\%) } \\
\text { Feminino: 135(73\%) }\end{array}$ \\
Idade na consulta & $50 \pm 12$ anos \\
Idade de experimentação & $15 \pm 5$ anos* \\
Idade de consumo regular & $18 \pm 7$ anos \\
Tentativas de abandono prévias & $3 \pm 2 * *$ \\
Outros fumantes em casa & $86(46 \%)$ \\
Escala de Fargeström & $6 \pm 2$ \\
Comorbidades físicas & $116(62 \%)$ \\
Comorbidades psiquiátricas & Sim: $44(24 \%)$ \\
Tratamento clínico & Sim: $113(61 \%)$ \\
Tratamento psiquiátrico & Sim: 67 (36\%) \\
\hline
\end{tabular}

* Dados disponíveis para 184 fumantes;

** dados relativos a 155 fumantes. 
A idade de primeiro contato com o tabaco, disponível para 184 pessoas, foi em média, $15 \pm 5$ anos, com variação entre sete e trinta e seis anos. A idade do começo do uso regular de cigarros foi de $18 \pm 7$ anos, variando entre sete e sessenta e um anos.

Tentativas prévias de parada foram relatadas por 155 indivíduos (83\%). O número médio de tentativas de cessação do fumo para esses 155 fumantes foi $3 \pm 2$ vezes, e o tempo médio decorrido entre a última tentativa e a consulta foi $54 \pm 78$ meses. A média do maior tempo sem fumar nessas tentativas foi $7 \pm 21$ meses.

$\mathrm{O}$ valor médio da escala de Fargeström para o grupo foi de $6 \pm 2$, variando entre zero e dez. Baseando-se nessa escala o grau de dependência à nicotina dos fumantes atendidos pode ser classificado em: ${ }^{6}$

- Muito baixo (Fargeström 0-2): 11 (6\%)

- Baixo (Fargeström 3-4): 20 (11\%)

- Médio (Fargeström 5): 33 (18\%)

- Alto (Fargeström 6-7): 76 (41\%)

- Muito alto (Fargeström 8-10): 46 (25\%)

Pelo menos alguma queixa física foi relatada por $77 \%$ dos fumantes, a mais frequente sendo tosse com expectoração (52\%). A presença de co-morbidades clínicas foi relatada por $116(62 \%)$ dos atendidos (Tabela 2). Dezesseis indivíduos negaram co-morbidades, mas faziam uso crônico de algum tipo de medicamento como, por exemplo, diuréticos e anti-inflamatórios. Se adicionarmos essas 16 pessoas aos 116 iniciais, $71 \%$ dos atendidos podem ser considerados portadores de alguma co-morbidade física. Esse subgrupo era composto por 35 homens e 97 mulheres, com idade média $52 \pm 11$ anos. O valor médio da escala de Fargeström para tais indivíduos foi $6 \pm 2$. Entre os fumantes atendidos, as moléstias mais frequientemente referidas foram hipertensão arterial $(55 ; 27 \%)$, dislipidemia $(29 ; 16 \%)$ e diabetes mellitus $(26 ; 14 \%)$. Entre as co-morbidades relatadas, em 15 casos (8\%) tratavam-se de moléstias diretamente atribuídas ao fumo, tais como obstrução arterial periférica (2), insuficiência coronariana (2), doença pulmonar obstrutiva crônica (6), perda óssea mandibular (1), câncer de orofaringe (1) e câncer de pulmão (1). Uso regular de medicamentos de natureza clínica tais como anti-hipertensivos, anti-lipêmicos, hipoglicemiantes orais, antiinflamatórios e vitaminas, foi relatado por $112(60 \%)$ pessoas.
Co-morbidades psiquiátricas foram relatadas por $44(24 \%)$ dos entrevistados. Um número adicional de pessoas (26) relatava o uso regular de algum psicofármaco como, por exemplo, ansiolíticos e antidepressivos, apesar de negar doença psiquiátrica. Quando somamos os últimos 26 pacientes aos 44 iniciais, obtemos um total de 70 fumantes (38\%), que podem ser classificados como portadores de algum distúrbio psiquiátrico. Esse sub-grupo foi composto por 11 homens $(16 \%)$ e 59 mulheres (84\%), com idade média $51 \pm 11$ anos, e escala de Fargeström de $6 \pm 2$. Entre os 44 pacientes que referiram a presença de co-morbidades psiquiátricas as mais comuns foram depressão (32; $17 \%)$, esquizofrenia $(4 ; 2 \%)$, transtorno bipolar $(3 ; 2 \%)$ e etilismo $(3 ; 2 \%)$. Seis outros indivíduos, não classificados como portadores de co-morbidades psiquiátricas atuais, relataram tratamento medicamentoso para depressão no passado. No tocante aos 67 fumantes que referiam emprego de algum tipo de medicação psiquiátrica, a maioria tomava anti-depressivos (48; 26\%), em especial fluoxetina, e diazepínicos (40;22\%). Outras medicações informadas foram anti-psicóticos $(5 ; 3 \%)$ e anticonvulsivantes $(2 ; 1 \%)$. Com freqüência foram observadas associações do uso de duas ou mais medicações psiquiátricas pelo mesmo indivíduo.

\section{Tabela 2}

Co-morbidades clínicas referidas por 116 fumantes em consulta inicial no programa de abandono de tabagismo.

$\begin{array}{lrc}\text { Hipertensão arterial sistêmica } & 55 & (47 \%) \\ \text { Dislipidemia } & 29 & (25 \%) \\ \text { Diabetes mellitus } & 26 & (22 \%) \\ \text { Cardiopatias } & 12 & (10 \%) \\ \text { SIDA } & 8 & (7 \%) \\ \text { Tireoidopatias } & 8 & (7 \%) \\ \text { Neoplasias } & 5 & (4 \%) \\ \text { Osteoporose } & 5 & (4 \%) \\ \text { Fibromialgia reumática } & 5 & (4 \%) \\ \text { Gastrite } & 4 & (3 \%) \\ \text { Arteriopatia periférica } & 2 & (2 \%) \\ \text { AVC prévio } & 2 & (2 \%) \\ \text { Outras condições diversas } & 22 & (19 \%)\end{array}$




\section{Discussão}

A população atendida no ambulatório no período foi composta majoritariamente por mulheres. Achados semelhantes já foram descritos por outros serviços de atendimento a tabagistas em anos recentes. ${ }^{7,8,9}$ Essa maior procura pode ser explicada pelo fato das mulheres exibirem maiores cuidados com a saúde do que os homens. Além disso, muitas delas não tinham compromissos profissionais, o que facilitava o comparecimento às consultas. Paradoxalmente, é sabido que a maioria dos fumantes são homens. ${ }^{10}$ Esses dados indicam a necessidade de campanhas de conscientização acerca dos males do cigarro dirigidas particularmente ao sexo masculino. Ainda, programas de abandono do tabagismo devem ser disponibilizados em horários que não conflitem com as atividades profissionais da maioria dos trabalhadores.

A idade média dos fumantes girou em torno dos 50 anos. Essa média é similar àquelas já relatadas por outros serviços de atendimento a tabagistas. ${ }^{7,8,9}$ Ainda que haja grande variação em torno das idades médias, os resultados sugerem que a maioria dos fumantes toma a decisão de procurar um serviço especializado num momento da maturidade, quando já houve conscientização dos riscos potenciais à saúde. Esse dado pode também significar que a procura por auxílio especializado ocorre apenas após diversas tentativas solitárias de abandono frustradas.

Uma porção expressiva dos indivíduos (41\%) referiu busca pelo auxílio de natureza espontânea. Esse resultado está de acordo com o fato de a maioria dos fumantes desejar abandonar o uso dos cigarros. ${ }^{1,4}$

A idade média do primeiro contato com os cigarros e do início de consumo regular encontradas aqui, estão perfeitamente de acordo com os dados da literatura. É reconhecido que a maioria dos fumantes inicia-se no vício com idade inferior aos 20 anos. ${ }^{11,12}$ Além disso, mais de $95 \%$ dos indivíduos atendidos referiam o consumo de cigarros de papel apenas, sugerindo que outras formas de consumo de tabaco são hoje de menor importância no Brasil.

Mais de $80 \%$ dos fumantes referiram experiências prévias frustradas de abandono. Esse percentual está dentro da faixa observada em estudos recentes, que variou de 50,3\% até 81,3\%. ${ }^{7,8,9}$ Enorme variação foi observada quanto ao maior período sem uso de cigarros nessas tentativas, desde um ou dois dias até 15 anos. Esses dados mostram que a nicotina é droga de difícil abandono, a ocorrência de recaídas fazendo parte da história natural da dependência. ${ }^{4,11}$
A maioria dos fumantes (66\%) exibia dependência à nicotina de grau alto ou muito alto, o escore médio da escala de Fagerström sendo 6. O Ministério da Saúde do Brasil indica a introdução de terapia medicamentosa em fumantes com Fagerström igual ou superior a $5 .{ }^{13}$ Seguindo esse critério, $83 \%$ dos indivíduos atendidos teriam indicação do uso de medicamentos para parar de fumar, em conjunto, naturalmente, com intervenções cognitivo-comportamentais. Esses dados apontam para a necessidade de ampla e ininterrupta disponibilização de medicamentos, com eficácia comprovada na cessação do tabagismo, nos centros de tratamento especializados.

Quase a metade das pessoas atendidas informou haver outros fumantes em casa. Essa ocorrência diminui as chances de abandono, bem como demanda esforços para inclusão dos demais moradores fumantes no tratamento. ${ }^{14}$

Aproximadamente $70 \%$ dos fumantes atendidos relatavam co-morbidades físicas e/ou uso crônico de medicamentos, o que pode ser, pelo menos em parte, justificado pelo fato de quase $60 \%$ deles terem sido encaminhados a partir do próprio sistema de cuidados médicos do CSE-FMRP e HCRP. As co-morbidades mais comuns encontradas foram a hipertensão arterial, dislipidemias e o diabetes mellitus, todas condições de risco para o desenvolvimento de doenças cardiovasculares. Como o tabagismo é importante fator de risco cardiovascular, capaz de potencializar os demais citados, pode-se inferir a importância da disponibilização de intervenções anti-tabágicas eficazes para tais pacientes. Não menos importante é o fato de $8 \%$ dos atendidos que procuraram o programa já exibirem moléstias diversas diretamente atribuídas ao fumo. Vale ressaltar que a ocorrência de sintomas respiratórios em indivíduos tabagistas costuma ser elevada, mesmo naqueles com história de consumo de cigarros de curta duração. ${ }^{15}$

Quase um quarto dos fumantes atendidos relatava distúrbios psiquiátricos, e mais de um terço o uso de psico-fármacos. A associação de tabagismo e certos distúrbios psiquiátricos é bem reconhecida, em especial quadros depressivos e esquizofrenia. ${ }^{16}$ Nessa situação, os efeitos psicofarmacológicos associados à inalação de nicotina poderiam atenuar manifestações da própria doença psiquiátrica. Dentro desse contexto o abandono da dependência pode se mostrar muito difícil. ${ }^{4,16}$ Além disso, frequentemente, tais pacientes fazem uso de anti-psicóticos, o que pode levar a dificuldades extras para o manuseio da terapia farmacológica anti-tabágica. A alta prevalência de co-morbi- 
dades psiquiátricas em pacientes fumantes aponta para a necessidade da participação também de especialistas em saúde mental nos programas de abandono do fumo. Além da presença de psicólogos na coordenação de grupos de apoio, o acesso rápido a médicos especialistas em psiquiatria, para a dissipação de dúvidas terapêuticas e resolução de problemas agudos, é de importância fundamental.

O presente trabalho sofre de uma série de limitações, entre elas seu caráter retrospectivo e o fato das entrevistas terem sido feitas por entrevistadores distintos em ocasiões diversas. Além disso, as informações relativas a co-morbidades físicas e uso de medicações foram obtidas a partir do relato dos pacientes, mas sem uso de uma planilha com opções padronizadas. Do mesmo modo, a presença de comorbidades psiquiátricas baseou-se unicamente na informação dos fumantes atendidos. É muito provável que, se fosse aplicado um instrumento padronizado de investigação de ansiedade e depressão, à semelhança do que foi feito em estudo anterior, a incidência desse distúrbio na presente amostra fosse maior. ${ }^{8}$

A criação de centros de referência para tratamento de fumantes é importante medida de saúde pública, e os dados aqui expostos podem servir como embasamento das necessidades a serem supridas por novos serviços que estejam prestes a se instalar.

A criação de centros de referência para tratamento de fumantes é, reconhecidamente, importante medida de saúde pública. Os dados aqui expostos servem como indicação do perfil da clientela a ser atendida por novos programas de tratamento de fumantes, que estejam prestes a se instalar em unidades de atendimento primário à saúde no Brasil.

\begin{abstract}
Objective: To describe demographic and clinical features of smokers who had looked for care in a smoking cessation specialized center. Methods: It has been retrospectively analyzed the data from standardized clinical forms, completed in the moment of the first consultation of smokers from the Outpatient Service of Support to Smokers of the "Centro de Saúde Escola da Faculdade de Medicina de Ribeirão Preto da Universidade de São Paulo", for a period of 18 months. Results: The outpatient clinic received 186 new smokers (135 women), with a mean age of $50 \pm 12$ years old, in this time. Most of the smokers (59\%) came from other medical outpatient services, and smoking of industrialized cigarettes only was reported by $96 \%$ of them. The beginning age of cigarettes regular consumption was $18 \pm 7$ years. Previous frustrated smoking cessation experiences were reported by $83 \%$ of the smokers, and the mean number of attempts was $3 \pm 2$ years. Most of the subjects $(66 \%)$ showed nicotine addiction of high and very high degree. The mean score of the Fargeström nicotine dependence test, for the whole group, was $6 \pm 2$. Physical co-morbidities were reported by $62 \%$ and psychiatric ones by $24 \%$ of the subjects, particularly depression cases (17\%). Conclusions: The present data is a starting point, regarding the needs to be fulfilled by new smoking cessation services to be installed in medium size towns from Brazil and abroad.
\end{abstract}

Key words: Smoking. Tobacco Dependence. Nicotine Dependence. Tobacco Use Cessation. Epidemiology, Descriptive.

\section{Referências Bibliográficas}

1. Benowitz NL, Brunetta PG. Smoking Hazards and Cessation. In: Mason RJ, Broaddus VC, Martin TR, King Jr. TE, Schraufnagel DE, Murray JF, Nadel JA. Murray \& Nadel's Textbook of Respiratory Medicine. 5th ed. Philadelphia: Saunders Elservier; 2010. p. 968-84.

2. Wünsch Filho V, Mirra AP, López RVM, Antunes LF. Tabagismo e câncer no Brasil: evidências e perspectivas. Rev Bras Epidemiol 2010; 13: 175-87.

3. Centers for disease control and prevention. Smoking-atributable mortality, morbidity and economic costs. Disponível em http:// apps.nccd.cdc.gov/sammec/. Acessado em 21/12/2011.
4. Reichert J, de Araújo AJ, Gonçalves CMC, Godoy I, Chatkin JM, Sales MPU et al. Diretrizes para cessação do tabagismo 2008. J Bras Pneumol 2008; 34: 845-80.

5. Faulkner MA, Lenz TL, Stading JA. Cost-effectiveness of smoking cessation and the implications for COPD. Int $\mathrm{J}$ Chron Obstruct Pulmon Dis 2006; 1: 279-87.

6. Meneses-Gaya IC, Zuardi AW, Loureiro SR, Crippa JA. Psychometric properties of the Fagerström Test for Nicotine Dependence. J Bras Pneumol 2009; 35: 73-82.

7. Sales MPU, Figueiredo MRF, Oliveira MI, Castro HN. Ambulatório de apoio ao tabagista no Ceará: perfil dos pacientes e fatores associados ao sucesso terapêutico. J Bras Pneumol 2006; 32: 410-7. 
8. Santos SR, Gonçalves MS, Leitão Filho FSS, Jardim JR. Profile of smokers seeking a smoking cessation program. J Bras Pneumol 2008; 34: 695-701.

9. Caram LMO, Ferrari R, Tanni SE, Coelho LS, Godoy I, Martin RSS et al. Characteristics of smokers enrolled in a public smoking cessation program. J Bras Pneumol 2009; 35: 98085.

10. Malta DC, Moura EC, Silva SA, Oliveira PP, Costa e Silva VL. Prevalence of smoking among adults residing in the Federal District of Brasília and in the state capitals of Brazil, 2008. J Bras Pneumol 2010; 36: 527-34.

11. Kirchenchtejn C, Chatkin JM. Dependência da nicotina. J Bras Pneumol 2004; 30 (Supl 2): S11-S18.

12. Ruffino Netto $A$, Caron-Ruffino $M$, Passos ADC. Hábito de fumar entre acadêmicos ligados à área de saúde. Alguns aspectos econômicos. Medicina (Ribeirão Preto) 1989; 22: 95-104.
13. Brasil. Ministério da Saúde. Instituto Nacional de Câncer INCA. Abordagem e Tratamento do Fumante - Consenso 2001. Rio de Janeiro: INCA, 2001, 38 p.

14. Doréa AJP, Botelho C. Fatores dificultadores da cessação do tabagismo. J Bras Pneumol 2004; 30 (Supl 2): S41-S46.

15. Ruffino Netto $A$, Caron-Ruffino $M$, Costa-Passos AD, Silva CA. Tabagismo e sintomas/doenças do aparelho respiratório entre acadêmicos ligados à área de saúde - Ribeirão Preto (SP). J Pneumol 1989; 15: 8-10.

16. Rondina RC, Gorayeb R, Botelho C. Psychological characteristics associated with tobacco smoking behavior. J Bras Pneumol 2007; 33: 592-601. 